\title{
Leishmaniasis cutánea, a propósito de un caso
}

Cutaneous leishmaniasis, a purpose of a case

Rodas Mosquera Juan Enrique', Valdivieso Díaz Angie Cecibel²

VOLUMEN 37 | No 1 | ABRIL 2019

FECHA DE RECEPCIÓN: : 04/02/2019 FECHA DE APROBACIÓN: 17/04/2019 FECHA PUBLICACIÓN: 30/04/2019

1. Libre ejercicio

2. Hospital José Carrasco Arteaga

\begin{tabular}{c|l} 
Caso & $\begin{array}{l}\text { Clinical } \\
\text { Case }\end{array}$ \\
Clínico &
\end{tabular}

Correspondencia:

juanen90ahotmail.com

Dirección:

Miguel Cordero Crespo y Rafael Fajardo

Código Postal: 010207

Teléfonos:

(07)2856666 - 0991467611

Cuenca - Ecuador

\section{RESUMEN}

La Leishmaniasis es una enfermedad parasitaria catalogada como emergente y sin control debido al cambio en el perfil epidemiológico por el surgimiento de nuevos focos y urbanización del ciclo de transmisión. Se describe el caso de un adolescente de la Comunidad Dos Ríos, del Cantón Taisha, quien presentó varias lesiones ulceradas, confirmándose diagnóstico de Leishmaniasis Cutánea mediante estudio histológico, iniciándose tratamiento con sales de antimonio pentavalentes, logrando una resolución progresiva y paulatina de las lesiones. Destacándose la importancia del diagnóstico temprano, tratamiento supervisado, y seguimiento para prevenir complicaciones.

Palabras clave: leishmaniasis cutánea, enfermedades cutáneas parasitarias/complicaciones, población rural, adolescente, gluconato de sodio antimonio.

\section{ABSTRACT}

Leishmaniasis is a parasitic, emergent and uncontrolled disease due to the change in the epidemiological profile for the appearance of new outbreaks and urbanization of the transmission cycle. A case of a 15-years-old adolescent, who is resident of the Community Dos Ríos located on the Taisha Canton, was described. The patient presented ulcerated lesions, confirming the diagnosis of Cutaneous Leishmaniasis through the histological study of the lesions, and starting intramuscular treatment with pentavalent antimony salts observing healing with a progressive and gradual resolution of the lesions and emphasizing the importance of early diagnosis, supervised treatment, and monitoring to prevent complications.

Key words: leishmaniasis cutaneous, skin diseases parasitic/complications, rural population, adolescent, antimony sodium gluconate. 


\section{INTRODUCCION}

La Leishmaniasis es una enfermedad producida por un parásito protozoario intracelular obligado del género Leishmania [1], es una zoonosis transmitida por un insecto díptero hembra del género Lutzomya que se encuentra predominantemente en las zonas rurales de las Américas y del género Phlebotomus en el resto del mundo, teniendo como reservorios animales tanto domésticos como silvestres incluido el hombre [2].

El insecto díptero, del género Lutzomya, Ilamado también "manta blanca" es un mosquito que mide de 1.5 a $3 \mathrm{~mm}$ que vuela bajo y de forma silenciosa a manera de saltos o brincos, habita de preferencia en lugares húmedos, oscuros donde exista abundante vegetación, característica importante para su sobrevivencia, descansa durante el día y vuela al atardecer, las hembras son las únicas hematófagas y más activas en horario entre $18 \mathrm{~h} 00$ y las $6 \mathrm{hO0}[3]$.

Existen cuatro tipos clínicos de Leishmaniasis: cutánea (viejo y nuevo mundo), cutánea difusa, mucocutánea y visceral [4], esta patología se caracteriza por presentar lesiones mutilantes y/o deformantes causando daño psicológico, aislamiento y perdida de la capacidad productiva de los afectados, constituyéndose en un grave problema de salud pública debido al trastorno físico, psicológico, socio cultural y económico de los pacientes, así como por los altos costos que genera su tratamiento [5].

\section{EPIDEMIOLOGIA}

La Organización Mundial de la Salud considera a la Leishmaniasis como una de las seis enfermedades tropicales más importantes que afecta a poblaciones rurales de escasos recursos, enfatizando que los factores sociales, ambientales y climatológicos influyen en la epidemiología de la enfermedad, es endémica en 98 países del mundo y se estima que 310 millones de personas están en riesgo de enfermar, se reporta que hay 14 millones de personas infectadas y cada año se registran aproximadamente una incidencia de 1.3 millones de los cuales 300 mil son viscerales y entre 20,000 y 30,000 personas mueren por esta causa [6-7].

En el Ecuador representa un problema de salud pública ya que afecta a personas vulnerables de sectores rurales que habitan en condiciones propi- cias para su transmisión y que tienen difícil acceso a transporte y medicación, fue reportada por primera vez en 1920, en la provincia de Esmeraldas (Leishmaniasis Cutánea), y en 1924 se reportó el primer caso de Leishmaniasis Mucocutánea y desde el año 2005 es una enfermedad de notificación obligatoria [8].

Según el Ministerio de Salud Pública durante el periodo del año 2000 al 2012 se han reportado casos en 23 de las 24 provincias del país, exceptuando Galápagos; con un promedio de 1,537 casos anuales y una tasa de incidencia que oscila de 6.14 a 19.15 por 100,000 habitantes [9]. La provincia con más casos reportados fue Pichincha con el $20.1 \%$ seguida de Esmeraldas con 16.4\%, Manabí con $12.8 \%$, Morona Santiago con $9 \%$, Orellana con $7 \%$ [9].

De acuerdo a características demográficas en el año 2014 se menciona que el grupo etario más afectado en Ecuador comprende edades de 20 a 49 años [10].

\section{CASO CLÍNICO}

Se presenta el caso clínico de un paciente de 15 años de edad, de sexo masculino, residente en la zona rural de la Comunidad Dos Ríos del Cantón Taisha de la Provincia de Morona Santiago, en Ecuador; de ocupación agricultor. Sin antecedentes de importancia. Destacándose que su vivienda carece de servicios básicos de infraestructura y su localización es en medio de exuberante vegetación.

Acude al puesto de salud de su comunidad con cuadro clínico de dos meses aproximadamente de evolución; presentando lesión inicial de $2.5 \mathrm{~cm}$. cerca de la articulación de la muñeca de mano derecha y caracterizado como una pápula, ovalada levemente pruriginosa, que se torna de color rojizo, cuya superficie se cubre de escamas furfuráceas; que cambió paulatinamente a un nódulo eritematoso, pruriginoso e indoloro con exudado seropurulento, dando formación a una costra adherida. Al mes del desprendimiento de la costra se observó lesión única, ulcerada de tejido granuloso, de forma circular, de bordes regulares elevados de tono violáceo edematoso y bien definidos de aproximadamente $3.5 \mathrm{~cm}$. de diámetro cubierto por tejido necrótico y exudado seropurulento en su interior (Imagen $\mathrm{N}^{\circ} 1$ ). Una vez ulcerada la lesión descrita 
anteriormente, en un lapso de 8 días, se presentó una segunda lesión de $2 \mathrm{~cm}$. de diámetro en región de carpo de mano ipsilateral, en forma de pápula, que evolucionó a nódulo pruriginoso, indoloro, con presencia de tejido necrótico (Imagen $\mathrm{N}^{\circ} 1$ ). El paciente buscó ayuda médica, por la falta de respuesta de tratamientos convencionales.

\section{Imagen $\mathrm{N}^{\circ} 1$}

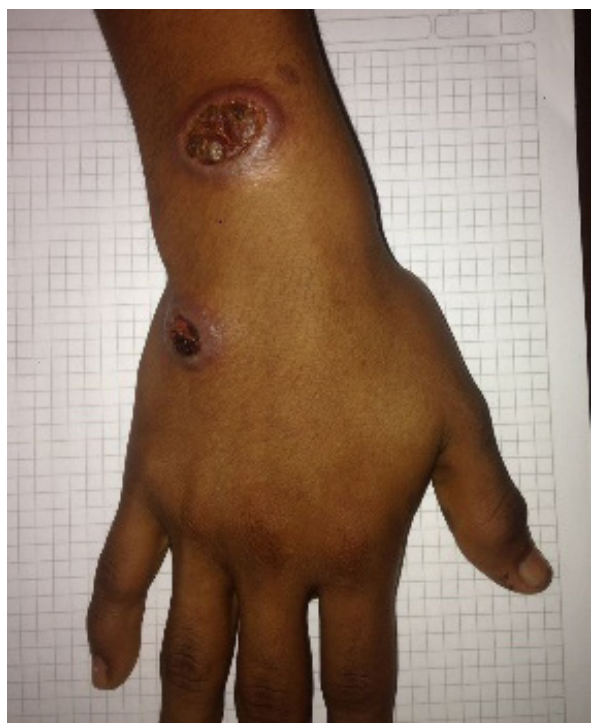

Lesiones nodulares con ulceración central y bordes elevados y definidos.

Por la procedencia del paciente, el cuadro clínico y las lesiones de la piel se realiza presunción diagnóstica de Leishmaniasis Cutánea, se envía a Hospital de Taisha para que se realice exámenes complementarios, entre los cuales se solicita examen histológico de las lesiones (Imagen $\mathrm{N}^{\circ} 2$ ).

\section{Imagen $\mathrm{N}^{\circ} 2$}

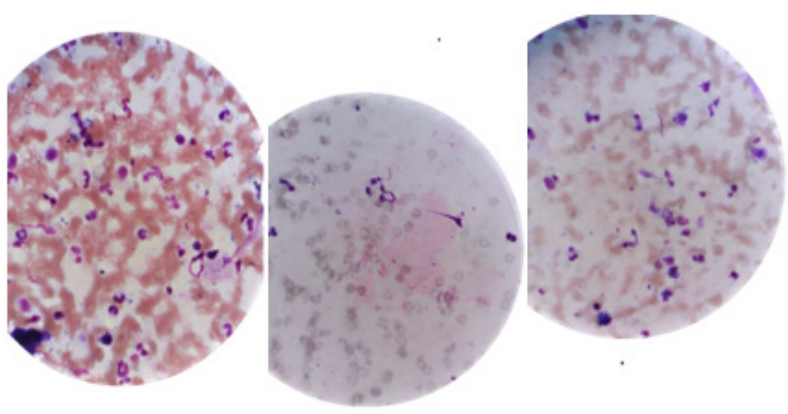

Leishmaniasis cutánea, formas parasitarias intracelulares
El procedimiento para el diagnóstico de Leishmaniasis Cutánea en el paciente, fue el frotis directo, que consiste en el raspado del borde interno de la úlcera con el borde romo de una lanceta, luego el material obtenido se extiende en forma suave sobre una lámina portaobjetos, limpia y debidamente rotulada, se deja secar la muestra a temperatura ambiente, se fija con metanol y se deja secar. Luego se tiñe las láminas con colorante de Giemsa al $10 \%$ en solución amortiguada de fosfatos con un pH de 7.2 durante 10 minutos, luego se observa al microscopio de luz con un aumento de 1,000; el objetivo es la inmersión para buscar los amastigotes que pueden encontrarse intra o extracelularmente. El examen fue positivo al encontrarse más de un amastigote [11]. (Imagen $\mathrm{N}^{\circ} 2$ )

Con diagnóstico confirmado se inicia esquema de tratamiento con sales de antimoniato de meglumina, calculando la dosis a $70 \mathrm{mg} / \mathrm{kg} /$ día por el peso del paciente correspondió a una dosis de 2 ampollas de $5 \mathrm{ml}$ diarias por vía intramuscular por 20 días.

Actualmente el antimoniato de meglumina está indicado para el tratamiento de Leishmaniasis Cutánea y mucocutánea y Leishmaniasis visceral (Kala azar). El mecanismo de acción de los antimoniales pentavalentes es transformase a antimonio trivalente que sería el responsable de la actividad protozoaria al inhibir diferentes enzimas del protozoo inhibiendo la glucólisis como la oxidación de los ácidos grasos e induce la disminución de la biosíntesis energética del amastigote, una de las fases en el ciclo vital de la Leishmania [12].

Se logran identificar 3 lesiones más, durante el curso de tratamiento, localizadas en línea axilar anterior derecha, indolora de $2 \mathrm{~cm}$ de diámetro (Imagen $\mathrm{N}^{\circ} 3$ ), en muslo lateral izquierdo de $2 \mathrm{~cm}$ (Imagen $\mathrm{N}^{\circ} 4$ ) y muslo posterior de $3 \mathrm{~cm}$ aproximadamente, redondas de bordes elevados e indoloras levemente pruriginosas. 


\section{Imagen $\mathrm{N}^{\circ} 3$}

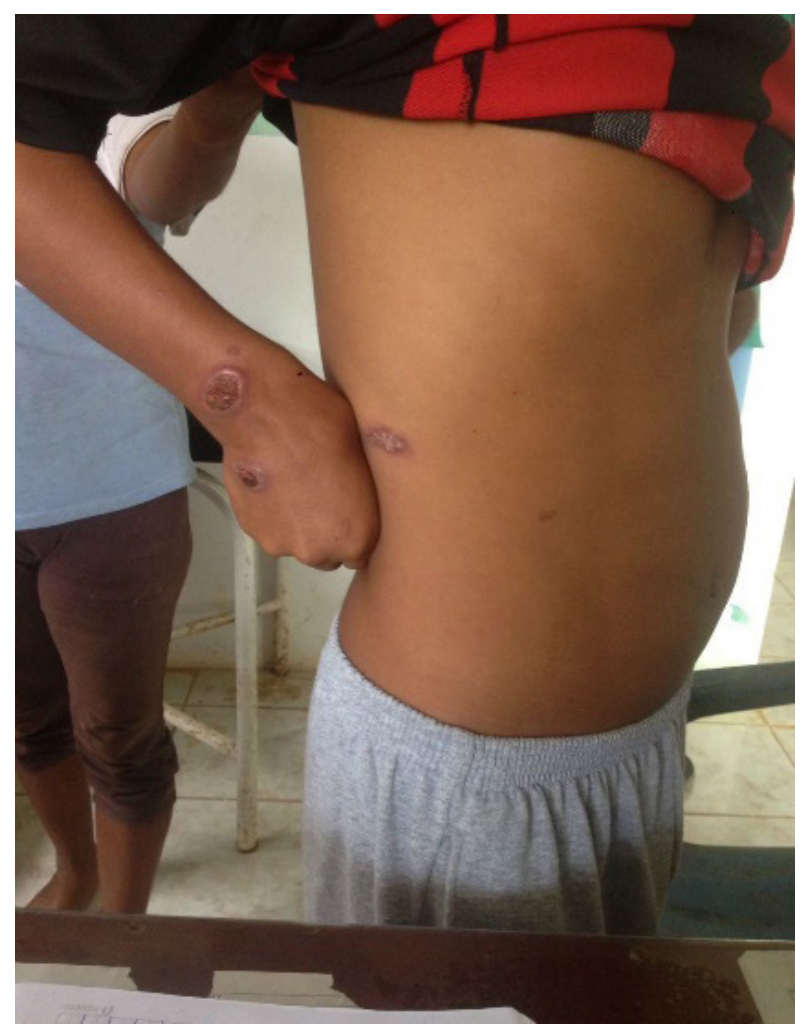

Lesión en línea axilar anterior derecha.

$$
\text { Imagen } \mathrm{N}^{\circ} 4
$$

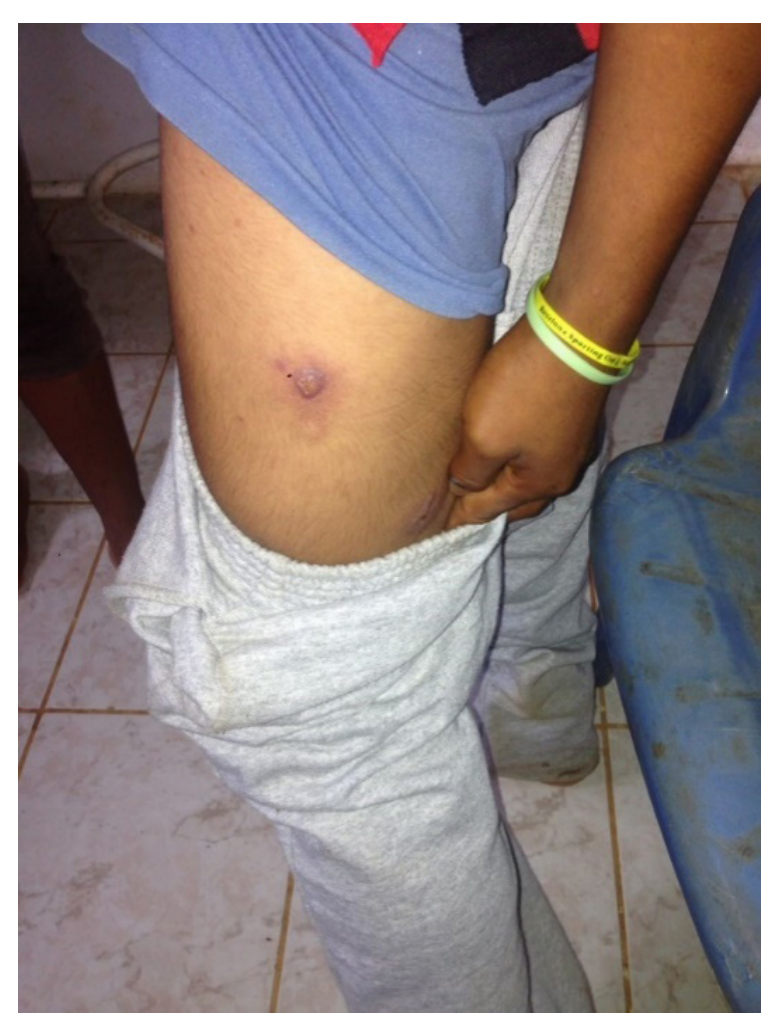

Lesión en muslo lateral
Se realizó control a los diez días de tratamiento observándose que el exudado desaparece, iniciando la regresión y reparación de la lesión a partir del centro hacia el exterior (Imagen $\mathrm{N}^{\circ} 5$ ).

\section{Imagen $\mathrm{N}^{\circ} 5$}

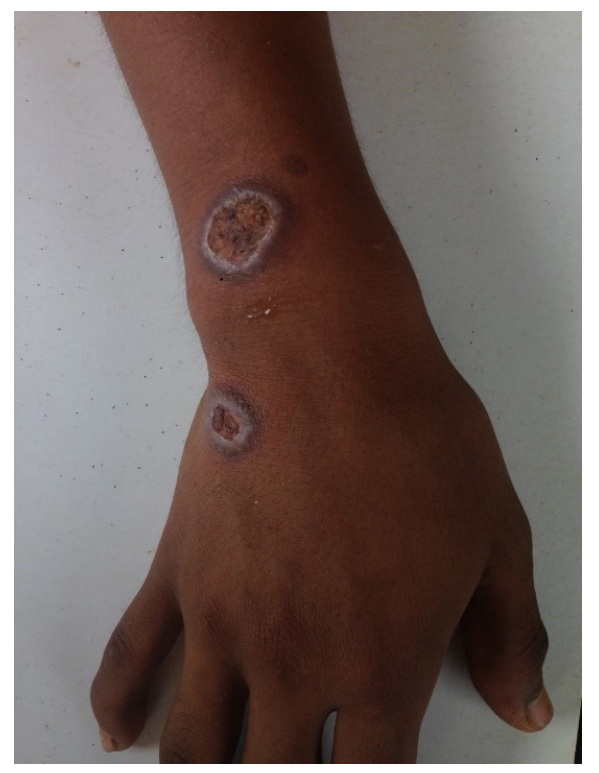

Exudado ausente, reparación de la lesión.

A los 20 días de finalizar el tratamiento se observó la formación de una cicatriz lisa, deprimida, hipocrómica e inestética (Imagen $\mathrm{N}^{\circ} 6$ ).

\section{Imagen $\mathrm{N}^{\circ} 6$}

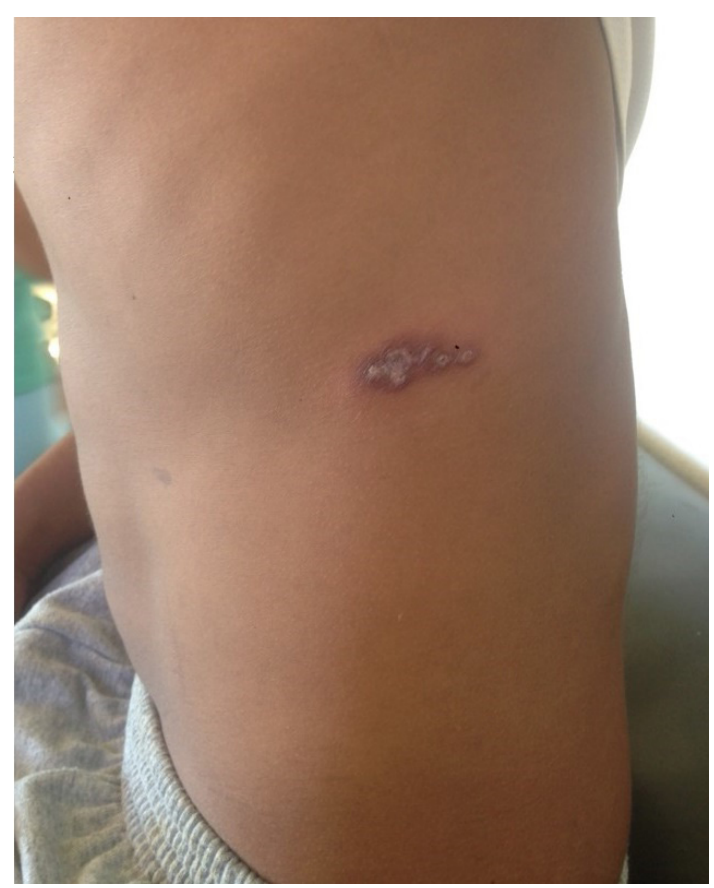




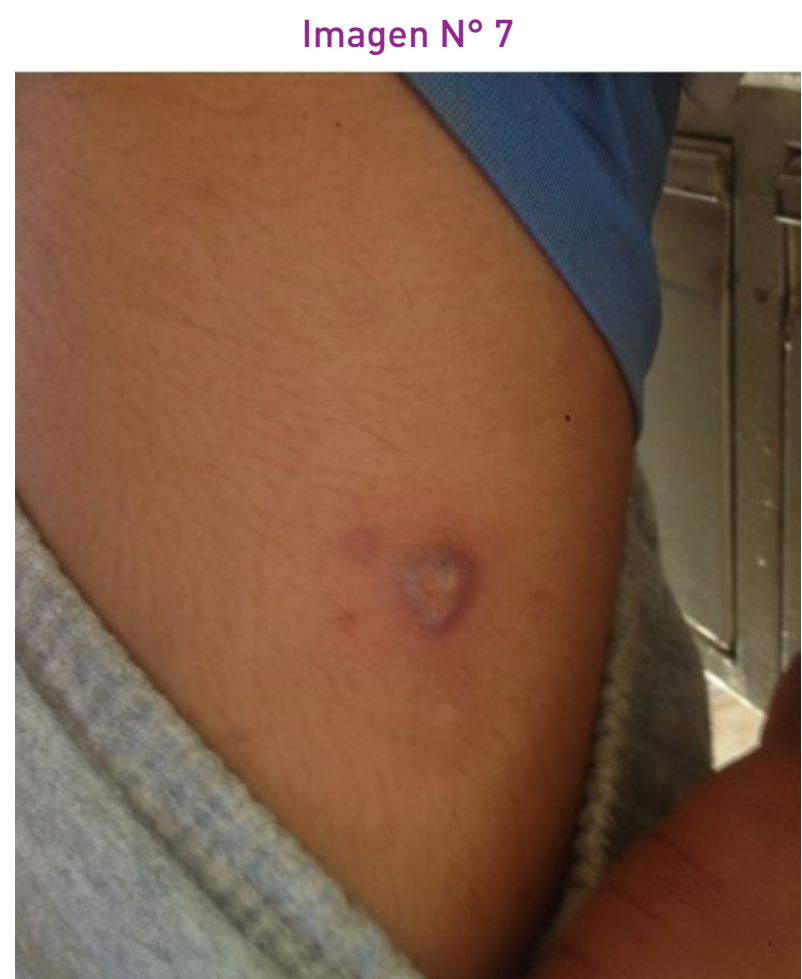

Imagen $N^{\circ} 8$

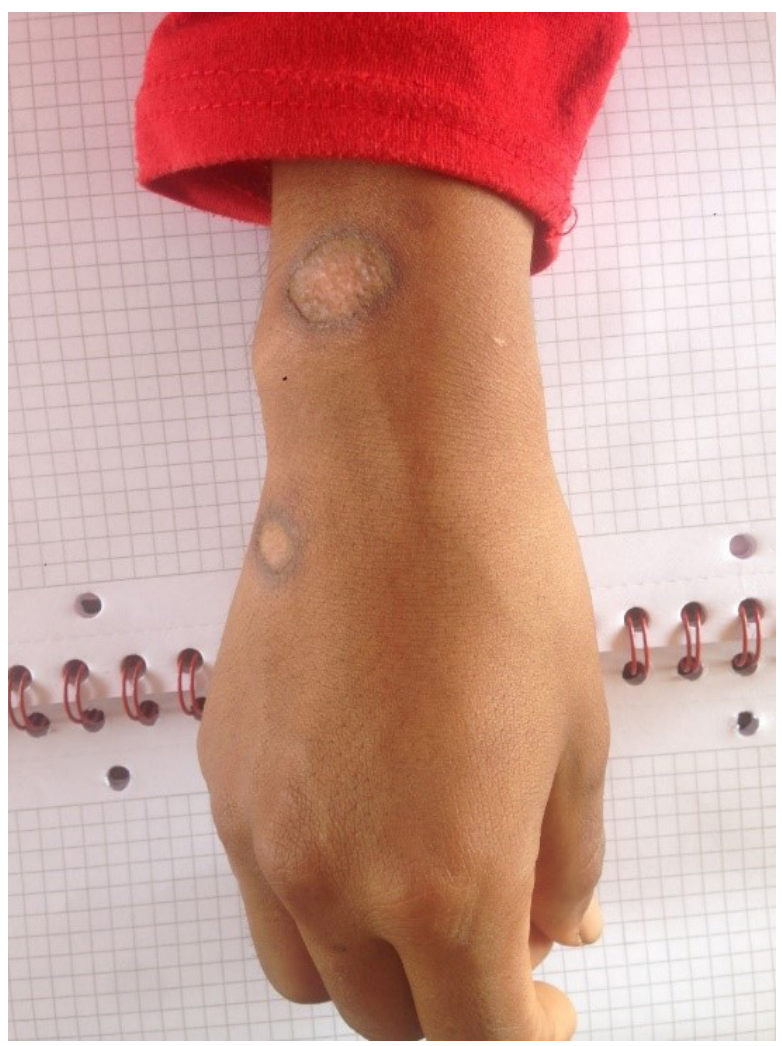

Las imagenes $\mathrm{N}^{\circ} 6 \mathrm{~N}^{\circ} 7 \mathrm{~N}^{\circ} 8$ muestran cicatrices hipocrómica en las diferentes localizaciones.

\section{DISCUSIÓN}

El presente caso pone de manifiesto las dificultades del personal médico al realizar el año de Medicatura Rural en zonas boscosas de difícil acceso y con cambios ambientales constantes que provocan como reporta la OPS variaciones en los ciclos de transmisión, los reservorios, los flebótomos vectores, las manifestaciones clínicas y la respuesta al tratamiento [13].

La incidencia de la Leishmaniasis ha aumentado en la última década, principalmente en Latinoamérica, debiéndose esto a la permanencia y actividades realizadas por el hombre en regiones amazónicas [14]. La Leishmaniasis Cutánea, presenta un amplio espectro clínico que dificulta su diagnóstico, por lo que es importante recopilar criterios epidemiológicos, ocupacionales, clínicos y de laboratorio, teniendo en cuenta los distintos diagnósticos diferenciales como: micosis profunda, xantomas, linfomas cutáneos y lepra lepromatosa Además lesiones ulcerosas: úlceras traumáticas, úlceras vasculares y linfáticas, piógenas, esporotricosis fija y linfangítica, paracoccidioidomicosis, TBC cutánea, úlceras por mico bacterias atípicas, pioderma gangrenoso y tumores malignos ulcerados, e infecciones por cocos gran positivos. Lesiones papulosas, nodulares $o$ en placas: picaduras de insecto con formación de granuloma, sarcoidosis, psoriasis. Lesiones verrugosas: cromomicosis, tuberculosis verrugosa, histoplasmosis, lobomicosis, carcinomas espinocelulares. Formas linfangíticas: esporotricosis, úlceras por micobacterias atípicas [15].

No existe ningún tratamiento óptimo [16-18], no obstante la OMS, sugiere el uso de drogas antimoniales pentavalentes como el Estibogluconato Sódico o Antimonio de Meglumina, que han sido durante décadas el único tratamiento disponible y que fue utilizado en el presente caso. La administración puede ser mediante vía intramuscular o vía parenteral, con tasas de curación de hasta el $93 \%$ en la Leishmaniasis Cutánea del nuevo mundo o con tasas de 55.6 a $92 \%$ del viejo mundo. Debiéndose tener en cuenta sus efectos adversos que habitualmente son reversibles como: dolor músculo esquelético, nefrotoxicidad, cardiotoxicidad, pancreatitis y discrasis sanguíneas [19]. En los últimos años han aumentado los casos de fracaso terapéutico por resistencia parasitaria a los fármacos o por inmunosupresión del paciente [20]. 
Existen otras variedades terapéuticas como: pentamidina, que se asocia con efectos secundarios como anorexia, náuseas, mareos, hipotensión, necrosis en el lugar de punción cuando se administran a dosis elevadas, por este motivo se reserva para casos refractarios [21]. Paramomicina tópica (Leschutan $\circledast$ ) usada en casos sin riesgo de afectación mucosa. Anfotericina $\mathrm{B}$ que posee efectividad moderada a dosis de $0.5-1 \mathrm{mg} / \mathrm{kg}$ por vía parenteral hasta 8 semanas [22]. Miltefosina por vía oral a dosis de $2.5 \mathrm{mg} / \mathrm{kg}$ al día [23], otros posibles tratamientos son la termoterapia y crioterapia, el Imiquimod Tópico [24, 25]. Pentoxifilina en asociación a N-metilglucamina efectivo en casos de mala respuesta a los antimoniales.

Es importante instaurar un tratamiento temprano, aunque la epitelización y el aplanamiento total de las lesiones de todas formas dejaran cicatrices notables [26].

En la actualidad en Colombia el tratamiento de la Leishmaniasis Cutánea es sistémico, y el uso de alternativas terapéuticas estará condicionado a tratamiento médico y a condiciones específicos como embarazo. El medicamento de primera elección son las sales de antimonio pentavalente que se realizan de forma ambulatoria, sistémica y prolongada bajo la supervisión del personal de salud para garantizar la adherencia al tratamiento. La vía de administración más empleada es la intramuscular (glúteos, vasos mayores y deltoides) el tratamiento de segunda línea se basa en Paramomicina y Anfotericina B [27].

En Brasil, el tratamiento de primera línea son los antimoniales pentavalentes por vía endovenosa, debido a que por vía intramuscular es muy doloroso. La vía intralesional es muy común sobre todo en las formas nodulares y las formas ulcerativas únicas o múltiples siempre y cuando el paciente no tenga más de cuatro lesiones y no exista evidencia de celulitis o linfangitis y de segunda línea la Anfotericina $B$ en caso de falla terapéutica a 2 series completas y dosis adecuada de tratamiento con antimoniales pentavalentes en los casos de lesión mucosa leve a moderada y de inicio en los casos de lesión mucosa severa [28].

En Perú, como primera línea de tratamiento se administra un antimonial pentavalente de tipo Estibogluconato sódico o Antimoniato de Meglumina, una sola dosis fraccionada por vía intravenosa, diluido en suero fisiológico, en caso de presentar fracaso al tratamiento luego de 2 meses de haber completado el primer ciclo de tratamiento, se repite el esquema de antimonial pentavalente por un segundo ciclo a la misma dosis por 20 días. Si luego de 2 meses persiste el fracaso terapéutico se opta por el esquema de segunda línea, Anfotericina $B$ a dosis de $0.5 \mathrm{mg}$ a $1.0 / \mathrm{kg} /$ día hasta acumular dosis máxima de 1.5 a 2.0 gramos por el periodo de tratamiento [29].

En Bolivia, en la fase cutánea el medicamento utilizado es el Antimoniato de Meglumina (Glucanti$\mathrm{me}$ ), ampolla de $5 \mathrm{ml}$. La dosis utilizada es de 20 $\mathrm{mg} / \mathrm{Kg}$ por día y por 20 días. Durante la fase mucosa, el medicamento de elección es la Anfotericina $\mathrm{B}[30]$.

Finalmente, las actividades de promoción, prevención y control recomendadas en zonas endémicas son orientadas a interrumpir la transmisión y disminuir la probabilidad de que una persona expuesta al riesgo de infección desarrolle la enfermedad. Evitar la picadura del mosquito mediante la utilización de medidas de protección personal como: uso de mosquiteros impregnados con insecticidas piretroides de acción residual, repelentes, destrucción de reservorios, eliminación de aguas estancadas, uso de ropa gruesa y de mangas largas. Está en proceso de investigación una vacuna [31].

\section{CONCLUSIONES}

Paciente de 15 años procedente del sector rural de la provincia de Morona Santiago, luego de ser diagnosticado de Leishmaniasis Cutánea recibió tratamiento con sales de antimoniato pentavalentes bajo vigilancia supervisada presentando una evolución favorable con resolución de las lesiones dérmicas.

\section{RECOMENDACIONES}

Es relevante reportar la experiencia de la atención a este paciente ya que debe ser considerado como una alerta al sistema de vigilancia epidemiológica y de la formación del personal de salud; resaltando la importancia de la historia clínica, con énfasis en el motivo de consulta, anamnesis, sobre todo en datos epidemiológicos de residencia, procedencia, nexo epidemiológico, ocupación, condiciones de vivienda y ambiente laboral, además el examen físico, la evolución del cuadro clínico y la falta de res- 
puesta a tratamientos convencionales, así como el diagnóstico diferencial correcto. Por último, insistir en el diagnóstico precoz, tratamiento supervisado, y seguimiento de los pacientes para evitar complicaciones y lograr el bienestar de la población.

\section{ASPECTOS BIOÉTICOS}

El presente trabajo se realizó con el consentimiento informado de la paciente, con total confidencialidad de datos personales.

\section{INFORMACIÓN DE LOS AUTORES}

- Rodas Mosquera Juan Enrique. Médico. libre ejercicio.

ORCID: https://orcid.org/0000-0002-7174-4440

- Valdivieso Díaz Angie Cecibel. Médico. Hospital José Carrasco Arteaga.

ORCID: https://orcid.org/0000-0003-4783-2640

\section{CONTRIBUCIÓN DE LOS AUTORES}

Los dos autores declaran haber contribuido de forma similar en la idea, diseño del estudio, análisis, interpretación de información y han realizado conjuntamente la redacción de este trabajo.

\section{CONFLICTO DE INTERESES}

Los autores declaran no tener conflicto de intereses en la presente investigación.

\section{FUENTES DE FINANCIAMIENTO}

Autofinanciamiento

\section{REFERENCIAS BIBLIOGRAFICAS}

1. Murray, P. Capitol 82: Blood and Tissue Protozoa. Medical Microbiology. Elsevier Health Sciences. Philadelphia, 2013: 770.

2. Vélez, I., Robledo, S. Leishmaniasis. Fundamentos Básicos de Medicina, Microbiología de las infecciones Humanas. Corporación para investigaciones Biológicas. Medellín, 2007: 368-383.
3. Arenas, R. Leishmaniasis. Dermatología. Atlas, diagnóstico y tratamiento. McGraw-Hill. México, 2013: 562-568.

4. Vásconez-Zárate, N. Proceso, control y mejoramiento de la Salud Pública. Subproceso de Epidemiología. Manual de Normas para el control de Leishmaniasis. Ministerio de Salud Pública. Colombia, 2010.

5. Matute, N. Espinoza C, Alger J, Padgett D, López E, Zúñiga C. Caracterización clínico-epidemiológica de pacientes con leishmaniasis atendidos en el Hospital Escuela. Rev Med Hondur. Honduras, 2009: 77(6): 7-15.

6. Organización Mundial de la Salud, Serie de Informes técnicos 949. Control de las Leishmaniasis: informe de una reunión del Comité de expertos de la OMS sobre el Control de las Leishmaniasis, Ginebra, marzo 2010: 22-26.

7. Organización Panamericana de la Salud/ Organización Mundial de la Salud. Guía para el Desarrollo de Planes Integrados de Acción para la Prevención, Control y Eliminación de las Enfermedades Infecciosas Desatendidas. 2011.

8. Hashiguchi, Y, Vélez, L., Villegas, N., Mimori, T., Gómez, E., Kato, H.Acta Tropical. Leishmaniasis in Ecuador: comprehensive review and current status. Ecuador, 2017: 166, 299-315. doi: http://dx.doi.org/doi:10.1016/j. actatropica.2016.11.039

9. Ministerio de Salud Pública. Proyecto de vigilancia y control de vectores para la prevención de la transmisión de enfermedades metaxenicas en el Ecuador 2013-2017 Servicio Nacional de Control de enfermedades transmitidas por vectores artrópodos "SNEM" Guayaquil, marzo 2013.

10. Ministerio de Salud Pública del Ecuador. SIVEALERTA. Gaceta epidemiológica. Dirección 
Nacional de Vigilancia Epidemiológica. Ecuador, 2014: (2), 11. http://instituciones. msp.gob.ec/dps/snem/images/gaceta2.pdf

11. Ministerio de Salud Pública del Ecuador - Dirección General de promoción y prevención. Anexo 1. Leishmaniasis Técnicas y procedimientos en Leishmaniasis. Ecuador, octubre 2014.

12. Sáenz RE, Paz HM, Johnson CM, NarváezER, De Vásquez AM. Evaluación de efectividad y toxicidad del Pentostam $®$ y Glucantime ${ }^{\circledR}$ en el tratamiento de Leishmaniasis cutánea. Rev Med. Panamá, 1987:12:148-64.

13. Organización Mundial de la Salud. Leishmaniasis (2018). https://www.who.int/es/ news-room/fact-sheets/detail/leishmaniasis.

14. Alireza K, Alireza F. "Treatment of acute Old World cutaneous leishmaniasis: A systematic review of the randomized controlled trials". J Am Acad Dermatol 2007; 57: 2. 335. e1-335.e29.

15. Rincón M, Silva S. "Leishmaniasis cutánea diseminada: reporte de dos casos en Santander, Colombia". Rev Salud Pública. Colombia, 2009; 11(1): 145-150.

16. Reithinger R, Dujardin JC, Louzir H, Pirmez C, Alexancer B, Brooker S. Cutaneous leishmaniasis. Lancet Infect Dis. Estados Unidos, 2007;7: 581-96.

17. Minodier P, Parola P. Cutaneous leishmaniasis treatment. Travel Med Infect Dis. 2007;5: 150-8.

18. González U, Pinart M, Reveiz L, Alvar J. Interventions for old World cutaneous leishmaniasis.Cochrane Database Syst Rev. 2008: CD005067.

19. Lupi O, Bartlett B. "Tropical dermatology: Tropical disease caused by protozoa". J Am AcadDermatol 2009; 60: 897-925.
20. Croft SL, Sundar S, Fairlam AH. Drug resistance in leishmaniasis. Clin Microbiol Rev. 2006; 19:111-26.

21. Leder K, Weller PF. Treatment and prevention of leishmaniasis. USA, 2009 Disponible en www.uptodate.com.

22. Morrison B, Mendoza I, Delgado D, Reyes Jaimes O, Aranzazu N, Paniz Mondolfi AE. Diffuse (anergic) cutaneous leishmaniasis responding to amphotericin $\mathrm{B}$ : clinical dermatology concise report. Clin Exp Dermatol. 2010;35:116-20

23. Ordaz-Farias A, Muñoz-Garza FZ, SevillaGonzález FK, Arana-Guajardo A, OcampoCandiani J, Treviño-Garza N, et al. Case report: transient success using prolonged treatment with miltefosine for a patient with diffuse cutaneous leishmaniasis infected with LeishmaniaMexicana. Am J Trop Med Hyg. México, 2013;88:153-6.

24. Khatami A, Firooz A, Gorouhi F, Dowlati Y. Treatment of acute old world cutaneous leishmaniasis: a systematic review of the randomized controlled trials. J Am Acad Dermatol. 2007;57(2): 335.e1-29.

25. Dirección Nacional de Servicios Académicos Virtuales. Sede Bogotá DNSAV. Leishmaniasis. Colombia, 2001. Disponible en: www.virtual.unal.edu/co/.../cap9- 5c.ht

26. Báfica A, Oliveira F, Freitas $L$ a R, Nascimento EG, Barral A. American cutaneous leishmaniasis unresponsive to antimonial drugs: successful treatment using combination of $\mathrm{N}$-methilglucamineantimoniate plus pentoxifylline. Int J Dermatol. 2003;42: 203-7.

27. Ministerio de la Protección Social República de Colombia. Dirección General de Salud Pública.Instituto Nacional de Salud, Guía de Atención Clínica Integral del Paciente con Leishmaniasis. Bogotá 2010. 25- 
27. Disponible en www.minsalud.gov. $\mathrm{co} / \ldots /$ Salud $\% 20 \mathrm{Pública} / . . . /$ Clinica $\% 20$ Leishmaniasis.

28. Barral-Netto M, Badaro R, Barral A, Carvalho E. Imunologia da leishmaniose tegumentar. Revista da Sociedad Brasileira de Medicina Tropical. 1986; 19:173-91.

29. Alan J. Magill. Cutaneous Leishmaniasis in the returning Traveler. Infect Dis Clin N A. (2005); 19:241 - 266.

30. Hernan Bermudez P, Ernesto Rojas C. Leishmaniasis tegumentaria en Cochabamba y Bolivia.Editorial Moca. Bolivia, 2002: 7-43.

31. Arenas, R. Leishmaniasis.Mc Graw. Hill. 5ta edición Atlas, diagnóstico y tratamiento. México, 2013: 562-568. 